\title{
Los procesos de alcoholización en el Chaco boliviano
}

\section{Resumen}

El presente es un trabajo de investigación etnográfica en el campo de la salud. Tiene como objetivo determinar las representaciones y las prácticas que los ava-guaraníes del Chaco boliviano tienen con respecto al alcohol introducido por los karai. Para eso partí del modo de ser guaraní, "conociendo" el uso y el consumo actual de bebidas fermentadas tradicionales (chicha) que tiene este grupo étnico. Por otro lado "reconocí» el uso y el consumo problemático de alcohol y la repercusión de este problema en comunidades ava-guaraníes cordilleranas, abordándolo desde la prevalencia y las representaciones de los comunarios. Por último, desde la zona Ava en la cual me ubiqué, confronté ambos "conocimientos" desde las prácticas de los ava-guaraníes.

\section{Summary}

An ethnographic research paper is presented, which aims to determine representations and practices of the Ava-Guarani people of the Bolivian Chaco regarding alcohol, introduced by the Karai. The Guarani "way of life" is taken as a starting point, "acknowledging" present use and consumption of traditional fermented beverages in this ethnic group. Besides, there is a "recognition" of alcohol use and problematic use and its consequences in Ava-Guarani communities, by addressing prevalence and community members representations. Both "understandings" were then analysed.
Autor

Fabrizio Martínez Dibarboure

Doctor en Antropología (Universidad de la República, Udelar). Magíster en Ciencias Humanas (Udelar). Profesor de Filosofía (Instituto de Profesores Artigas). Especialista en Enfermería en Salud Mental y Psiquiatría (Udelar) y licenciado en Enfermería (Udelar). Hospital Vilardebó.

Correspondencia: martinezgrundel@gmail.com

\section{Palabras clave}

Antropología y salud

Ava-guaraníes

Karai

Chicha

Alcohol

\section{Key words}

Anthropology and health

Ava-Guarani

Karai

Chicha

Alcohol 
* Realizada en el marco de la maestría en Ciencias Humanas, cursada en la Facultad de Humanidades y Ciencias de la Educación de la Universidad de la República (Uruguay).

** Actualmente es el «denominativo con el que los guaraníes del Chaco boliviano designan a la gente extraña, foránea, blanca». Antiguamente este término se utilizaba entre los guaraníes para designar a sus «profetas», dado que «los karai eran líderes carismáticos, muchas veces itinerantes, que actuaban sobre todo en períodos de crisis. Eran ellos, como conciencia crítica del grupo, quienes percibían el mal en la tierra y luchaban contra este.»

*** Una de las etnias guaraníes que habitan el Chaco boliviano (sudeste de Bolivia).

**** Utilicé la caligrafía chiriguana (Ivi significa 'tierra'), o ava-guaraní de Bolivia, que difiere en algunos aspectos de la utilizada por los grupos mbya-guaraní en Paraguay (yvy 'tierra').

$* * * * *$ Curt Unckel, Nimuendajú (1883-1945), fue un etnólogo germano-brasileño.

$* * * * * *$ Pues altera toda la gama de la constelación de relaciones sociales, como el antropólogo francés Claude Lévi-Strauss (1908-2009) definía a la persona.

$\star * * * * * *$ Pueblos indígenas que se encuentran en Brasil, Perú y Colombia.

\section{Introducción}

Esta pesquisa en antropología y salud ${ }^{*}, 1$ profundiza las representaciones y las prácticas que sobre el alcohol de los $k_{a r a i} i^{* *}, 2,3$ tienen los ava-guaraníes. ${ }^{* * *}$

A primera vista, podría parecer un tema ampliamente abordado, pero el antropólogo francés Marc Augé nos dice que «tal vez la etnología pueda, pues, ayudar a comprender lo que nos es demasiado familiar para que no nos resulte ajeno». ${ }^{4}$

Comenzando el viaje, en el año 1999, tuve la oportunidad de compartir un tiempo de mi vida con los habitantes de un pequeño poblado de origen guaraní, a unos veinticinco kilómetros al norte de Goya (provincia de Corrientes, Argentina). La vida transcurría en torno al río Paraná, lugar de encuentro para estos pobladores del río. En estos contextos se daba la búsqueda de la $¥ v \dot{t}$ Maraët, ${ }^{* * * *}$ 'la tierra sin mal', es decir, ese «lugar accesible a los vivos, un lugar adonde, «sin pasar por la prueba de la muerte», se podía ir en cuerpo y alma». ${ }^{5}$ De aquí se desprende que «ninguno de los etnólogos que, desde Nimuendajú,***** estudiaron a los guaraníes pudo dejar de subrayar la importancia que estos indios otorgaban a su vida religiosa». 5

En el año 2005, el Pbro. Walter Nacho Aguirre, sacerdote uruguayo misionero durante varios años en comunidades guaraníes, un paí miní, a quien los guaraníes «bautizaron» mburika ('burro'), nos invita a mi esposa (también licenciada en Enfermería) y a mí a conocer el Chaco boliviano, donde nos vinculamos con los ava-guaraníes. Con él fui aprendiendo, parafraseando al antropólogo inglés Tim Ingold, la pasión por la diferencia como fundamento de la reflexión antropológica.

Este breve aspecto autobiográfico me sirve de preámbulo para tratar de hacer confluir la Antropología Social con mis profesiones de base que apuntan a incentivar la reflexión filosófica sobre el hombre y sus cuidados.
Cuestiones que podrán parecer antagónicas, pero, como Homo viator, «el etnólogo se encuentra siempre de viaje, en la medida que es un viajero de lo interno». ${ }^{6}$

Como preámbulo, es necesario precisar que en los contextos de las relaciones económicas de las poblaciones indígenas el alcohol de los karai es introducido para servir de «moneda», como parte de un «salario», etc. ${ }^{5}$ Por lo tanto, comencé a vislumbrar cómo el consumo de alcohol, junto con la depresión que origina en el sistema nervioso central y las complicaciones orgánicas, puede llegar a ahogar los espacios comunitarios, promover la violencia familiar e impedir la apertura hacia el otro. También constaté la «despersonalización» $* * * * * *$ que producía, pues estos pobladores hablaban continuamente guaraní, pero cuando se emborrachaban se insultaban en castellano: se decían «indio de mierda». ${ }^{7}$

\section{Antecedentes}

En el ámbito de investigaciones científicas precedentes, con respecto al estudio del «problema» del alcohol quiero puntualizar que ya en el Primer Congreso Mundial de las Ciencias Antropológicas y Etnológicas, celebrado en Londres en 1934, se llegó a la evidencia de lo que «resultó ser un caso clásico de experimentación natural: una sola especie (Homo sapiens), una sola sustancia (el alcohol) y una gran diversidad de comportamientos».8

En lo que respecta a la prevalencia de este problema, «la tasa de alcohol varía entre los diferentes grupos de una misma etnia basado en características como la edad, el sexo o la religión» ${ }^{8}$ y también «se asocia a otros problemas como la violencia social, la persistencia de un estado general de salud deficitario y una alta tasa de suicidio entre algunos grupos como los kaiowá-guaraníes y los tikuna $»^{* * * * * * *,}, 8$ 
Contemporáneamente, estudios etnomédi$\cos ^{*},{ }^{9}$ realizados en Brasil con los kaingang del estado de Paraná, los maxacali de Minas Gerais y los bororo de Mato Grosso muestran un consumo elevado y generalizado de alcohol en población indígena. ${ }^{10}$

Las investigaciones en el área de antropología y salud** me han permitido acercarme al problema del consumo de alcohol desde otras miradas y acceder a otro tipo de abordaje.

Es así que «por intermedio de la pesquisa, fue posible percibir la diversidad de situaciones ligadas al consumo de bebidas alcohólicas, con el contexto en que se bebe, la intensidad del consumo, las variaciones en los estilos de beber y, principalmente, si la bebida constituía un problema para aquella comunidad». ${ }^{11}$

Asimismo, «el fenómeno del uso de bebidas alcohólicas entre los mbyá-guaraníes ${ }^{* * *}$ se sitúa en el interior de una cultura de contacto producida durante el proceso histórico de contacto interétnico. Al consumo de alcohol se agrega un conjunto de prácticas y significados que articulan las concepciones y el estilo tradicional del grupo y los elementos de la sociedad occidental incorporados a ese universo (alimentos, músicas, bebidas alcohólicas, etc.), formando una "cultura de beber" específica». ${ }^{12}$

En otro contexto, una investigación multidisciplinaria realizada por la Cruz Roja de Suiza y el Ministerio de Justicia de Bolivia en el año 2006 muestra que en Alto Parapetí (departamento de Santa Cruz, Bolivia) perviven relaciones laborales servidumbrales en 26 haciendas, donde existen al menos 10 comunidades indígenas «cautivas». A cambio de vivienda y alimentación, las familias trabajaban las tierras de los hacendados. Los patrones fían o venden con sobreprecio coca, alcohol, ropa usada, aceite y otros alimentos, lo cual genera una situación de endeudamiento permanente producto de una forma de explotación «paternalista» de mano de obra, situación que se da también en otras zonas de Latinoamérica. ${ }^{13,14}$ Los trabajadores no pueden salir de las propiedades en tanto no paguen sus cuentas acumuladas, las que pueden llegar a ser vitalicias e incluso heredarse. ${ }^{15}$

Aunque el abuso de alcohol en los indígenas de Bolivia no es un fenómeno nuevo, hay pocas investigaciones que lo aborden; no obstante, este problema ha sido estudiado en otras regiones de América Latina. ${ }^{8}, 10,16-20$

\section{Localización}

La investigación se llevó a cabo en dos comunidades ava-guaraníes pertenecientes a la capitanía de Kaaguasu, del municipio de Gutiérrez, provincia Cordillera, departamento de Santa Cruz, al sureste de Bolivia. Incluyó las comunidades de Ipitacito e Itaimbeguasu para estudiar las representaciones y las prácticas que los habitantes de cada una de ellas tienen sobre el alcohol introducido por los karai. (Figura 1)

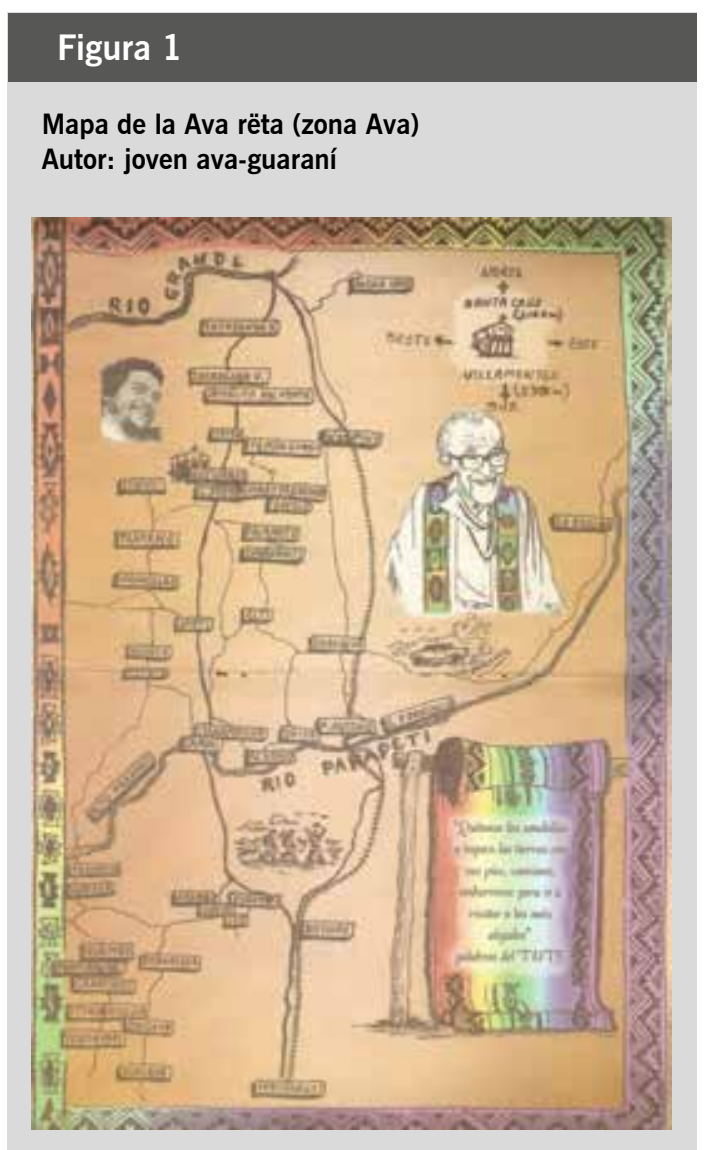

Trabajo original

* «La antropología médica es una de las más importantes especializaciones de las ciencias antropológicas. Nacida con el objetivo de orientar la investigación antropológica en torno a las formas con las que en las diferentes sociedades los seres humanos viven, representan y afrontan el sufrimiento y la enfermedad.»

** Antropología y salud, antropología de la enfermedad, antropología médica, etnomedicina, etc., si bien pueden utilizarse como sinónimos, implican concepciones diferentes.

*** Pueblo guaraní que habita en Argentina, Brasil y Paraguay. 
Así comencé a conocer desde una perspectiva emic* a estas comunidades, lo cual me permitió identificar los principales problemas que desde un punto de vista etic ${ }^{* *}$ observaba en el ámbito de los procesos de salud/enfermedad/ atención-prevención, pues, como nos refiere el antropólogo argentino Eduardo Menéndez, «a través de dichos procesos se expresa la sociedad o la cultura, pero sobre todo las particularidades de toda una serie de grupos que operan en una sociedad determinada. Dichos grupos tienen saberes similares, pero también desiguales y diferentes al menos respecto a algunos de los sufrimientos, de las enfermedades o de las estigmatizaciones que los afectan.» ${ }^{21}$ Esto me llevó a abordar a aquellos comunarios que se encontraban inmersos en un proceso de alcoholización, dado que este, en palabras de E. Menéndez, «refiere a los procesos económico-políticos y socioculturales que operan en una situación históricamente determinada para establecer las características dominantes del uso y consumo de alcohol (incluyendo el no uso y no consumo) por sujetos y conjuntos sociales». ${ }^{22}$

\section{Problemas, objetivos y métodos}

Me planteé preguntas como problemas a investigar: ¿Qué representa el alcohol de los karai para los ava-guaraníes? ¿Cómo se vinculan los ava-guaraníes con el alcohol de los karai? ¿Cómo afecta a los ava-guaraníes el alcohol de los karai?

Para poder estudiar dichos problemas, establecí como objetivo general determinar las representaciones y las prácticas que los ava-guaraníes tienen del alcohol de los $k a$ rai. A su vez, como objetivos específicos me planteé conocer el uso y el consumo actual de bebidas fermentadas tradicionales que tienen los ava-guaraníes; reconocer el uso y el consumo problemático de alcohol introducido por los karai en comunidades ava-guaraníes y su prevalencia a nivel de género y etapa del ciclo vital; y, por último, vincular estos «conocimientos».
Como método de investigación realicé un abordaje de tipo etnográfico. ${ }^{* * *}, 23$ Utilicé como técnicas de recolección de información la revisión bibliográfica, los antecedentes de investigaciones precedentes, las observaciones, los cuestionarios, las entrevistas y un diario de campo (donde día a día registré mis impresiones) para analizar representaciones, percepciones, estrategias y prácticas.

\section{Resultados}

Cada vez que llegaba a las comunidades cordilleranas me presentaba diciendo que venía de Uruguay y, por supuesto, refería que esa palabra provenía del guaraní con la tradicional traducción que solemos conferirle. Pero a una mujer le llamó la atención mi explicación; ella me pidió disculpas y me sugirió que el nombre de nuestro país también admite otros significados, pues uru podría ser 'gallina', tuguai podría ser 'cola' y la $y$ podría significar 'río' o 'agua'; ${ }^{24}$ por lo que «el paisito» podría significar 'cola de gallina' o 'cola de agua'. Mi sorpresa fue grande al escuchar esto, pero la mujer al verme confundido me dijo que no me preocupara, pues estas dos denominaciones de «cola» hacen referencia al fin y así hubiera seguido con esa incertidumbre si ella no me hubiera explicado que esos significados implicaban que nos hallábamos al final del territorio, es decir, donde finalizaba el dominio guaraní (mujer ava-guaraní, 53 años).

Diversos autores nos refieren que en el siglo XVI los guaraníes se expandían por regiones subtropicales de Argentina, Bolivia, Brasil, Paraguay y Uruguay. En Bolivia, el pueblo guaraní habitaba un extenso territorio conocido históricamente como «la Cordillera» por lo menos desde el siglo xv. Hoy en día los guaraníes se expanden por el oriente del territorio boliviano habitando en los departamentos de Santa Cruz, Tarija y Chuquisaca, diferenciándose los ava-guaraníes (habitantes del piedemonte andino) de los isoseños (habitantes del Chaco boliviano) y de los simba. ${ }^{3,25,26}$ Actualmente también 
encontramos a los guaraníes en el noreste de Argentina, en el centro-oeste de Brasil, en Paraguay y supieron estar en el norte de Uruguay. Es decir, abarcan geográficamente una gran extensión de la cuenca del Plata.

Durante dos años hice tres mboupara, es decir, 'visitas' al Chaco boliviano para realizar el trabajo de campo y estuve en total un semestre por la provincia Cordillera. La visita (mboupara) de enero de 2009 fue introductoria y me permitió definir en qué comunidades ava-guaraníes, de la capitanía de Kaaguasu, realizaría el trabajo de campo. Las estadías (setiembre de 2009, enero-febrero de 2010 y setiembre-noviembre de 2010) se realizaron de esa manera porque era lo que me permitían mis circunstancias familiares y laborales, pero me posibilitaron tomar «distancia»e ir decantando lo vivido.

$\mathrm{Al}$ abordar el ñande reko, el modo de ser guaraní, vi como un aspecto fundamental a considerar la fiesta del Arete Guasu en la que se celebra agradeciendo por el maíz, luego de la siembra y antes de la cosecha, y es la chicha uno de los elementos que constituyen esta festividad, donde se bebe en forma de kangüi (chicha dulce fermentada). De un tiempo a esta parte, esta fiesta guaraní por excelencia pasó a transformarse en carnaval, donde en vez de chicha se bebe el alcohol del karai. Observé que los guaraníes actuales caminan rumbo a

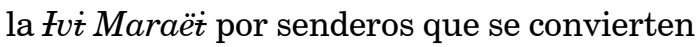
en «diabólicos» (pues dividen), como nos refería un abuelo ava-guaraní, pues el camino tiene luces y sombras, bien y mal, ${ }^{27}$ chicha y alcohol; hay que saber elegir para llegar a la Tierra-Sin-Mal, que es la comunidad ideal, al decir de un comunario, pues «con la chicha se reconocen; con el alcohol se desconocen y se pierden", al decir de otro comunario.

La chicha corresponde al «mundo de lo femenino", siendo un componente sim-bólico («lo que nos une») de vital importancia en la unidad familiar y comunitaria. Pues «la chicha es un símbolo de unión, donde está la chicha hay unión de la familia y la comunidad. Se comparte la chicha, que se saca de una tinaja y se reparte en el patio y nos ponemos a bailar» (varón ava-guaraní, 51 años). Antiguamente, al trabajo diario en el Chaco los hombres llevaban su tutumada, la chicha recién elaborada. (Figura 2)

\section{Figura 2}

Mujer ava-guaraní de Itaimbeguasu preparando chicha. (Foto: Fabrizio Martínez)

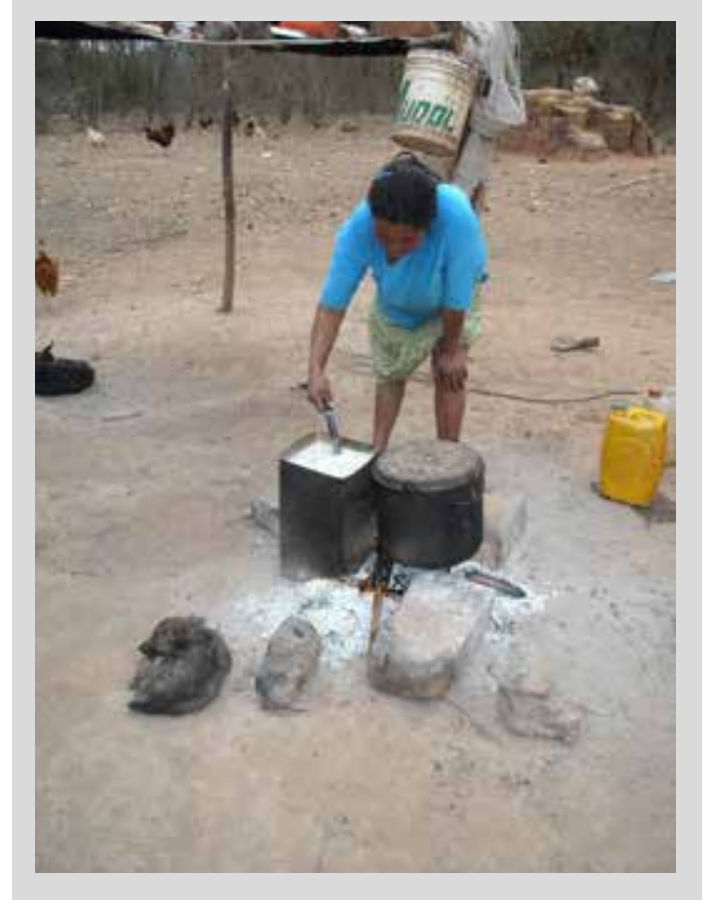

A lo largo de los siglos, desde la época del coloniaje español y agudizándose en la época republicana, particularmente a partir de Kuruyuki,* se ha ido creando un discurso karai en el cual se muestra al "guaraní flojo y borracho", representaciones que también ingresaron en la percepción de los guaraníes. En contrapartida, se ha ido creando otro tipo de discurso que tiene presupuestos «teóricos» diferentes del anterior. Esta dialéctica, a modo de ejemplo, la pude encontrar en una institución muy arraigada desde hace siglos en el Chaco boliviano, la Iglesia católica, donde ambos discursos tienen cabida y también sus predicadores.

Así, los ava-guaraníes existentes en las comunidades cordilleranas tienen percepciones
* En dicha batalla, acontecida en el Chaco boliviano el 28 de enero de 1892, cientos de jóvenes guerreros guaraníes fueron aniquilados por el ejército boliviano. 
ambivalentes de lo «bueno» y lo «malo», lo tradicional y lo moderno. Por ejemplo, las «creencias» más arraigadas expresan respeto y temor a los hechizos maléficos del mbaekuaa ('brujo'), y a la vez estas costumbres son combatidas e ironizadas en las comunidades. (Figura 3)

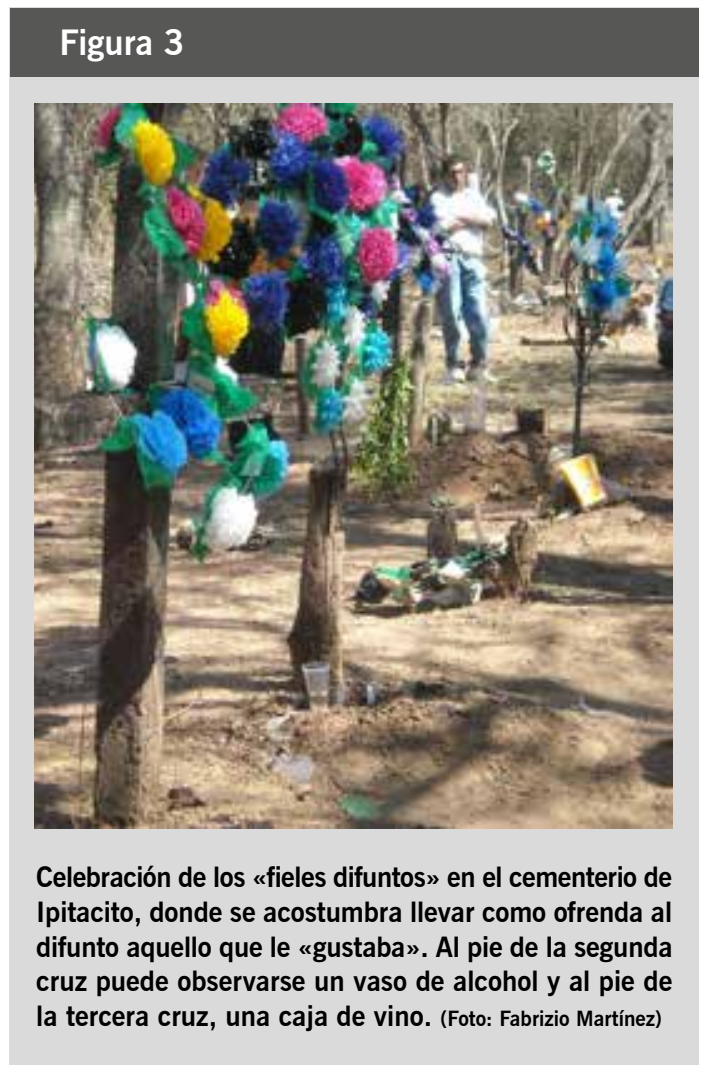

Otro tanto sucede con el alcohol. Todas las representaciones recogidas en las comunidades estudiadas, a la hora de categorizar y otorgar sentido al alcohol introducido por los karai, fueron unánimes al rechazarlo y condenarlo; sin embargo, a nivel de las prácticas, en lo referente a las estrategias de acción, los padecimientos y las consecuencias, observé disociaciones entre los discursos y las prácticas de los varones ava-guaraníes.

Se aprecia una alta prevalencia de consumo de alcohol en los varones a partir de los 15 años, cuando terminan los estudios primarios $\mathrm{y}$ al no tener otras «oportunidades» comienzan a trabajar en el Chaco; sobre todo en la comunidad de Itaimbeguasu, que al ser más apartada presenta menos posibilidades educativas. Así «entran» en el problema del abuso de esta sustancia, pues hoy llevan alcohol (de

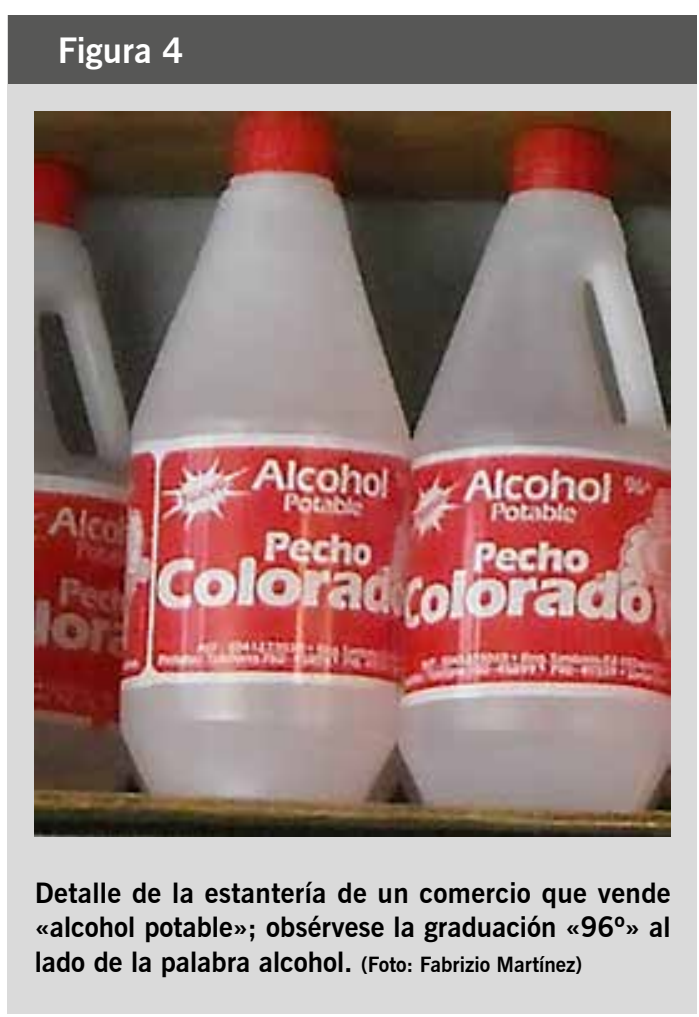

$96^{0}$ rebajado con agua), tabaco y coca y ya no más la tutumada. (Figura 4)

A modo de ejemplo, el mburuvicha guasu ('capitán grande') que me permitió estudiar el problema del alcohol en las dos comunidades de la capitanía de Kaaguasu, que dijo que este «era un flagelo de los karai», fue retirado de sus funciones por los propios comunarios, por «borracho y por aliarse con los patrones». Otros mburuvichas, que también condenan el alcohol del karai, hacen «alarde de su prestigio» al derramar cerveza, por ejemplo, frente a los demás y a la hora de decidir otros lo hacen por ellos. Otros comunarios condenaban el alcohol, pero luego se embriagaban y se ponían violentos, siendo particularmente las mujeres y los niños quienes recibían el castigo. 
Observé así que el alcoholismo corresponde al «mundo de lo masculino», siendo el alcohol de los karai un componente dia-bólico en la segmentación familiar y comunitaria. Si bien se pueden emborrachar con chicha, difícilmente haya mayores problemas; en cambio, las borracheras con alcohol originan muchas dificultades de violencia doméstica (tradicionalmente no sucede en la cultura guaraní) y otros problemas familiares y comunitarios. El guaraní que se emborracha con el alcohol del karai por lo general adopta una postura de desprecio hacia su familia y comunidad. Exterioriza todo el odio sufrido que tiene internalizado por siglos de opresión, y se comporta como un karai que, bajo los efectos del alcohol, denigra a los suyos, verbal y físicamente; deja de hablar en guaraní y habla en castellano cual si fuera un "patrón».

Hay que tener en cuenta, fundamentalmente, lo que piensan las mujeres en las comunidades. Todas, unánimemente, condenaron el consumo de alcohol y a la hora de las prácticas, salvo en las fiestas de navidad y en el Arete Guasu, suelen no beber alcohol.

Junto a la «visión» femenina, un factor de protección que tienen los ava-guaraníes, a tener en cuenta para contribuir a la prevención, es la unidad alrededor de la Asamblea del Pueblo Guaraní. Esta se inserta en la realidad actual del Estado Plurinacional de Bolivia defendiendo y promoviendo el modo de ser guaraní. Otro aspecto a considerar es la muy alta estima que los comunarios tienen hacia la institución escolar; de ahí que desde ella se podría seguir fomentando el valor de la cultura guaraní, sobre todo en relación con el consumo de su bebida tradicional como instrumento de unión e identidad.

Aparte del alcoholismo, producto de la transformación ${ }^{28}$ de los patrones de consumo en las comunidades, hay graves problemas de salud ligados a sus «costumbres». Por ejemplo, las enfermedades más prevalentes como el mal de Chagas (que afecta a toda la población) y los problemas respiratorios y gastrointestinales, que afectan particularmente a los niños, tienen mucho que ver con los estilos de vida y el entorno, ya que las viviendas inadecuadas (construcciones de paredes de tierra y techos de paja que son lugares propicios para cobijar la vinchuca, el vector del mal de Chagas), la carencia de servicios de agua potable y red de saneamiento básica y, por último, los hábitos de higiene (como un sencillo lavado de manos previo a la ingesta de alimentos) nos hacen recordar lo que estaba escrito en grandes carteles en las Postas de Salud: ñanoi vaera teko röisa ikavigue vae ndepope öi («la clave

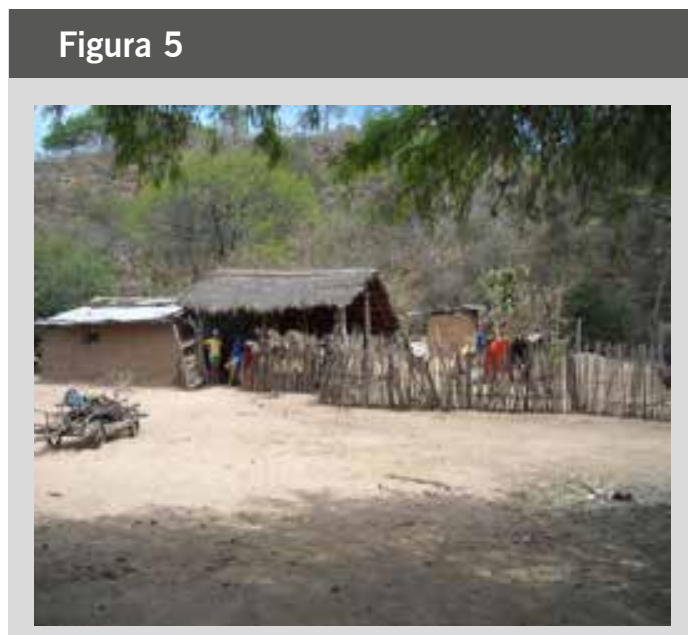

Vivienda de Itaimbeguasu. (Foto: Fabrizio Martínez)

para una buena salud está en tus manos»). (Figura 5)

Intentando confrontar lo anteriormente reseñado con mi experiencia asistencial en un hospital psiquiátrico público uruguayo, donde llegan diariamente personas con consumo problemático de alcohol, reflexionando desde una perspectiva filosófica y antropológica, pienso que la cuestión acerca de lo normal y lo patológico es algo que afecta no solamente el campo de las Ciencias de la Salud, sino que además la Filosofía y las Ciencias Sociales y Humanas pueden y deben reflexionar sobre ello.

$\mathrm{El}$ abordaje sanitario de una persona con problemas de consumo de alcohol lo tenemos que realizar desde un enfoque integral. Para esto la Antropología tiene mucho para aportarnos, dado que, como nos dice la antropóloga

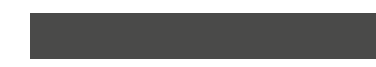


uruguaya Sonnia Romero, «la antropología (cuyo objeto científico es el ser humano y sus variedades diacrónicas y sincrónicas) contempla en toda su extensión la complejidad de la identidad que va desde lo biológico, lo psíquico, lo social a lo cultural, con un sentido muy denso para cada uno de estos dominios, ya que a su vez están compuestos por múltiples elementos, categorías y según una enorme cantidad de condicionantes, ambientales, históricas y otras». ${ }^{29}$

Muchas veces, por las dificultades propias de los equipos de salud (como de cualquier colectivo humano), se nos desdibujan estos insumos. Aunque parezca algo sencillo, un adecuado acercamiento y una mejora sustancial en la atención sanitaria implican la capacidad de escuchar. Eso me aportó, fundamentalmente, la aplicación del método etnográfico.

La observación y la escucha resultan estrategias imprescindibles de abordaje a la persona con problemas de consumo de alcohol. En la actualidad en el marco de una nueva Ley de Salud Mental, ${ }^{30}$ que apunta a un sistema de salud integrado, donde se disputan tantos «etnocentrismos» en juego, estos presupuestos pueden contribuir radicalmente a mejorar la atención de salud de la población, donde se comparte la labor desde el mismo entorno de la persona, su familia y su comunidad, que son tan necesarios para un verdadero cambio de paradigma.

Si partimos del concepto de que la atención ha de centrarse en la comunidad, la tendencia se centrará en el fomento de la Salud Mental y no tan solo en los aspectos curativos, ya sea en una población para evitar problemas de salud o bien en personas con problemas de adicciones, en las que queremos fomentar los aspectos «saludables» de su personalidad.

El marco de antropología y salud tiene mucho para aportarnos, pues nos fomenta a seguir interactuando con el otro de manera integral, y nos ayuda a entender las diferencias culturales con respecto a los cuidados humanos, la salud y la enfermedad entre, por ejemplo, los ava-guaraníes y otras culturas. Por lo tanto, estos supuestos nos ayudan a contribuir al desarrollo de la Enfermería en Salud Mental como una disciplina eminentemente social y antropológica.

\section{Referencias bibliográficas}

1. Martínez F. Karu Guasu. Entre encuentros y desencuentros: los Ava-Guaraní y su representación y práctica del alcohol de los karai. Disertación de maestría. Montevideo: Universidad de la República; 2013.

2. Chamorro G. Teología guaraní. Quito: Abya-Yala; 2004, p. 73.

3. Ortiz E. Toponimia guaraní del Chaco y Cordillera. Ensayo lingüístico, etnográfico y antropológico. Camiri: Teko Guaraní; 2004.

4. Achilli E. Investigar en Antropología Social: los desafíos de transmitir un oficio. Rosario: Laborde; 2005, p. 59.

5. Clastres H. La tierra sin mal. El profetismo tupí-guaraní. Buenos Aires: Ediciones del Sol; 1993.

6. Augé M. Por una antropología de la movilidad. Barcelona: Gedisa; 2007, p. 70.

7. Lévi-Strauss C. Antropología estructural. Buenos Aires: Eudeba; 1968.

8. Langdon E. L'abus d'alcool chez les peuples indigènes du Brésil: une évaluation comparative. Drogues, santé et société $2005 ;$ 4(1):15-52.

9. Pizza G. Antropología Médica: una propuesta de investigación. En: C. Lizón (ed.). Introducción a la Antropología Social y Cultural. Madrid: Akal; 2006, pp. 267-291.

10. Ferreira L, Coloma C. Approche intraculturelle destinée à réduire les dommages liés à la depéndance à l'alcool chez les Mbya-Guarani du Rio Grande do Sul, au Brésil. Drogues, santé et société 2005; 4(1):175-216.

11. De Oliveira M. A intervenção como um processo em construção: notas para a redução do uso de bebidas alcoólicas e alcoholismo entre os Kaingáng. En: E. Langdon, L. Garnelo (orgs). Saúde dos 
Povos Indígenas. Rio de Janeiro: ABA; 2004, pp. 69-87.

12. Ferreira L. O "fazer antropológico" em ações voltadas para a redução do uso abusivo de bebidas alcoólicas entre os Mbyá-Guarani, no Rio Grande do Sul. En: E. Langdon, L. Garnelo (orgs). Saúde dos Povos Indígenas. Rio de Janeiro: ABA; 2004, pp. 89-110.

13. Geffray C. Le modèle de l'exploitation paternaliste. En: Léna P, Geffray C, Araujo R. L'oppression paternaliste au Brésil. Paris: Lusotopie; 1996, pp. 153-159.

14. Bonilla O. O bom patrão e o inimigo voraz: predação e comércio na cosmología Paumari. Mana 2005; 11(1):41-66.

15. Bolivia. Viceministerio de Tierras. Unidad de Promoción Indígena y Campesina. La Paz: Boletín N. ${ }^{\circ} 82 ; 2008$.

16. Menéndez E. Morir de alcohol: saber y hegemonía médica. México: Alianza Editorial Mexicana; 1990.

17. Gutmann M. Etnicidad, alcohol y aculturación. Alteridades 2002; 12(23):19-34.

18. Huarcaya S. No os embriaguéis...: borracheras, identidad y conversión evangélica en Cacha, Ecuador. Quito: UASB, Abya-Yala; 2003.

19. Caux C. Histórias de cachaça e povos indígenas. Dissertação de Mestrado. Rio de Janeiro: Universidade Federal do Rio de Janeiro; 2011.
20. Perreault M. Alcool et Amérindiens: au-delà des stéréotypes. Drogues, santé et société 2005 ; 4(1):5-13.

21. Menéndez E. La parte negada de la cultura. Relativismo, diferencias y racismo. Barcelona: Bellaterra; 2002, p. 323.

22. Menéndez E, Di Pardo R. De algunos alcoholismos y algunos saberes. Atención primaria y proceso de alcoholización. México: CIESAS; 1996, p. 62.

23. Ramírez Hita S. La contribución del método etnográfico al registro del dato epidemiológico. Epidemiología sociocultural indígena quechua de la ciudad de Potosí. Salud Colectiva 2009; 5(1):63-85.

24. León de Santiago P, Nasini I, Ortiz García E. Diccionario guaraní-castellano y castellano-guaraní (1791). Camiri: Teko Guaraní; 1998.

25. Hirsch S. Construcción de las identidades en la frontera: el caso de los indígenas guaraníes de Argentina y Bolivia. La Plata: V Congreso Argentino de Antropología Social; 1997.

26. Chumiray G. Apuntes para la historia del pueblo guaraní en Bolivia. Camiri: Teko Guaraní; 2005.

27. Albó X, Libermann K, Godínez A, Pifarré F. Para comprender las culturas rurales en Bolivia. La Paz: Cipca; 1989.

28. Lévi-Strauss C. Raza y cultura. Madrid: Cátedra; 1993.

29. Romero S. Interrogaciones en torno a la cuestión «afro» y la discriminación en Uruguay: escenario para transformaciones identitarias. En: N. Guigou (comp. y org.). Trayectos antropológicos. Montevideo: Nordan; 2007, pp. 161-173.

30. Uruguay. Parlamento Nacional. Ley de Salud Mental N. ${ }^{\circ}$ 19.529. Disponible en: $<$ https://parlamento.gub.uy/documentosyleyes/leyes/ley/19529>.

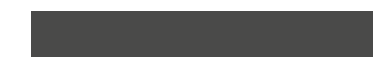

